\title{
Nanotechnology in radiation oncology: The need for implantable nano dosimeters for in-vivo real time measurements
}

\author{
Abdulhamid Chaikh1,2, Michael Beuve ${ }^{3,4}$, Jacques Balosso ${ }^{1,2}$ \\ ${ }^{I}$ Department of Radiation Oncology and Medical Physics, Grenoble University Hospital, Grenoble, France \\ ${ }^{2}$ Grenoble-Alpes University, Grenoble, France \\ ${ }^{3}$ Université de Lyon, Université Lyon 1, CNRS, Lyon, France \\ ${ }^{4}$ IN2P3, UMR 5822, IPNL, F-69622 Villeurbanne, France
}

Received February 27, 2015; Revised March 10, 2015; Accepted April 05, 2015; Published Online May 04, 2015

\section{Scientific $\mathcal{N o t e}$}

\begin{abstract}
Rapidly advancing technology provides successive generations of irradiation techniques and modalities of cancer treatment in radiation oncology. Most of these techniques are able to deliver higher doses per fraction than the standard 2 Gy per day. The complexity of these new techniques involves hundreds of parameters for the delivery of each beam making quality assurance increasingly demanding. A direct assessment of the "final product", namely the absorbed dose, would be extremely useful if easy to obtain. Thus, a real need exists for dosimeters able to provide direct and real time measurements within the target volume. Nanotechnology is a relatively new field, and in some ways raises new technological aspirations, especially in the field of medical applications for cancer treatment. In this paper we argue the need for an implantable "nano-dosimeter" based on nanotechnology to monitor the delivered dose, combining all the ideal features such a future tool should have for quality assurance in radiation oncology.
\end{abstract}

Keywords: Implantable Nano Dosimeters; in-vivo Real Time Measurements

\section{Radiation therapy modalities}

Radiation therapy techniques can be divided in four main types: external beam radiotherapy (EBRT), brachytherapy, intra-Operatory Radiation Therapy (IORT) and metabolic radiation therapy. In EBRT there are numerous different machines, all using compact linear accelerators applying several techniques, such as three-dimensional radiation therapy (3DRT), intensity-modulated radiation therapy (IMRT), volumetric-modulated arc therapy (VMAT), Tomotherapy, Stereotactic RT, etc. In addition to these X-ray based techniques one should also mention hadron therapy (mainly with protons and low atomic weight ions). Usually the total prescribed dose is delivered in several fractions, with a typical fraction of 2 Gy per day. Treatments often last well over one month with 5 fractions a week. The reason is the time necessary for the repair and regrowth of normal cells to allow an acceptable treatment tolerance for surrounding healthy tissues. Advanced techniques like VMAT may require shorter delivery times and can even be delivered as hypo-fractionated courses increasing the dose per fraction (above $2 \mathrm{~Gy}$ ) and decreasing the number of fractions. Stereotactic RT, for small volumes, is pushing this very far by delivering up to 6 to $25 \mathrm{~Gy}$ in few or even one unique fraction. Beside the economic aspects, the hypo-fractionated approach is justified by the fact that small doses per fraction are also less efficient on tumors. When anatomical and ballistic conditions restrict irradiation to almost only the tumor volume, a reduction of the fractionation is possible and improves the dose-effect on the tumor. However, this requires very high precision and demands ensuring the perfect achievement of each session by monitoring the deposited dose by an appropriate means, if possible in real time to allow immediate correction when needed.

\section{Dosimetry for quality assurance in irradiation therapy: state of the art}

To really improve quality assurance in radiation therapy, it is desirable to implement in-vivo real time measurement devices, such as miniature dosimeters. The objective is to ensure that the measured dose meets the prescribed dose. A change of $5 \%$ in the delivered dose can result in a 10 to $20 \%$ change in the local tumor control probability (TCP) and have up to a $30 \%$ impact in normal tissue complication probability (NTCP). ${ }^{1}$ However, the real clinical consequences of such deviations in the dose are modulated by the position of the dose level in the TCP and NTCP curves. ${ }^{2}$ Despite several 
existing technologies such as small ionization chambers, silicon diodes, metal-oxide semiconductor field effect transistor (MOSFET), thermo-luminescent dosimeter (TLD), optically stimulated luminescence (OSL), diamond and films, none are completely suitable for real time in-vivo measurements. ${ }^{3}$ The principle of detection of these dosimeters can be categorized into 4 types: air cavity, semiconductor, solid state and chemical, as shown in Figure 1. Ionization chambers are used to calibrate the linear accelerator and are not used as in-vivo dosimeters. The other types of dosimeters can be used for in-vivo measurements and can be classified according to the site of measurements, either external or internal:
Type (a): dosimeters for entry dose measurements such as diodes, TLD, films, etc. Since about a decade this type of "in-vivo dosimetry" has been used to measure the entry dose with dosimeters placed in the site where the beam penetrates the patient's skin. This method has been adapted for standard fractions of less than $2 \mathrm{~Gy}$ per session per beam.

Type (b): implantable dosimeters for measurements within the target volume including the tumor or organs at risk, such as MOSFET and solid state crystals $\left(\mathrm{GaN} ; \mathrm{Al}_{2} \mathrm{O}_{3} / \mathrm{C}\right)$. These so called, "implantable" dosimeters can be somehow introduced into the body either via natural cavities or, less frequently, directly into the tissues.

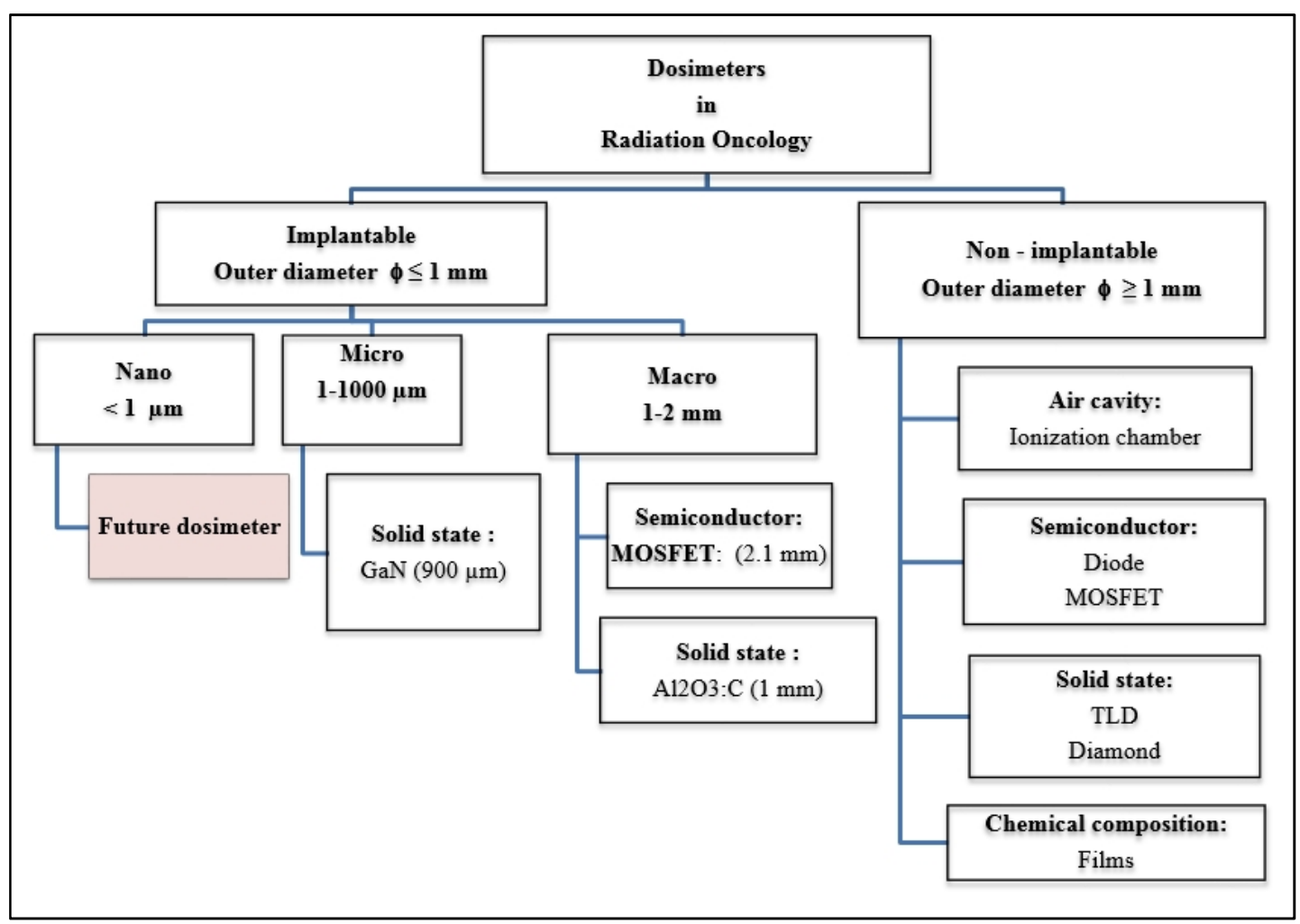

FIG. 1: Classification of dosimeters for dose measurements in radiation oncology.

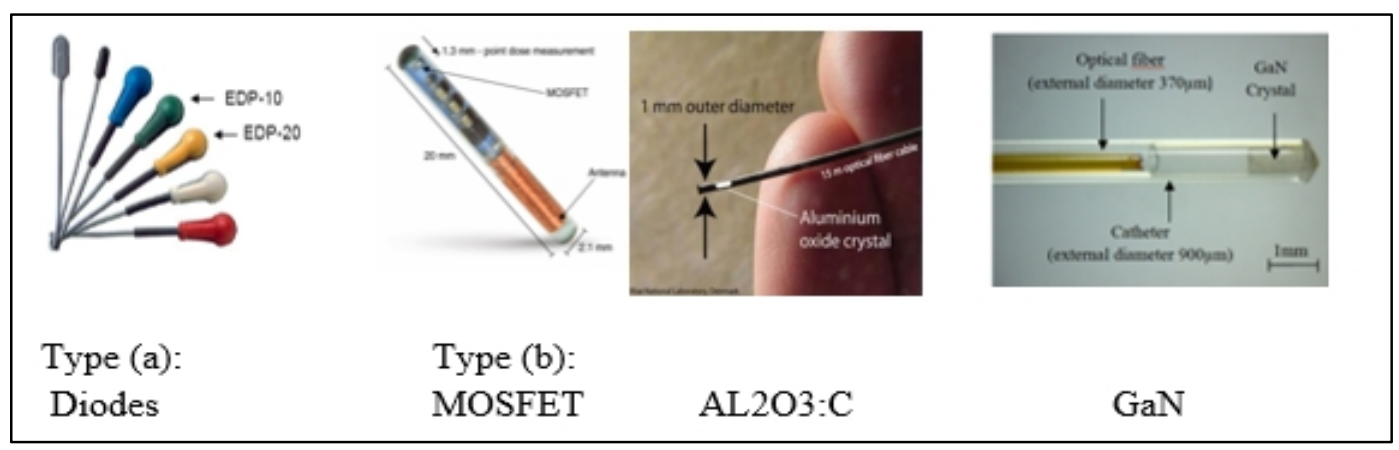

FIG. 2: Examples of external and implantable dosimeters for real time measurements. (a) The dosimeter from

(b) MOSFET from Sciel technologies® (Raleigh, NC), Al2O3/C from http://medicalphysicsweb.org and the GaN probe from Chaikh et al. 2014. 


\section{Implantable dosimeters based on macro/micro detectors for real time measurements}

The use of implantable dosimeters for monitoring the delivered dose has undergone some progress in the last few years. For example, the dose verification system from Sciel technologies ${ }^{\circledR}$ (Raleigh, NC) based on an implantable MOSFET dosimeter is already quite small $(20 \times 2.1 \mathrm{~mm}){ }^{4}$, Figure 1 . Recently OSL dosimeters such as carbon-doped aluminium oxide $\left(\mathrm{Al}_{2} \mathrm{O}_{3} / \mathrm{C}\right)$ have been improved and are proposed as implantable dosimeters for brachytherapy. ${ }^{5}$ More recently, a Gallium Nitride (GaN) based implantable dosimeter for real time measurements has been developed (Figure 2). ${ }^{6}$ The prototype includes a tiny $\mathrm{GaN}$ crystal $\left(<0.1 \mathrm{~mm}^{3}\right)$ coupled with an optical fiber of external diameter $0.9 \mathrm{~mm}$. GaN is a very efficient radioluminescent direct gap semiconductor. The GaN dosimeter is connected to a laptop equipped with a photo-multiplier. It offers interesting advantages for clinical use such as small size, radiation robustness and no chemical toxicity. The sensor has been calibrated but as yet few clinical studies have been performed.

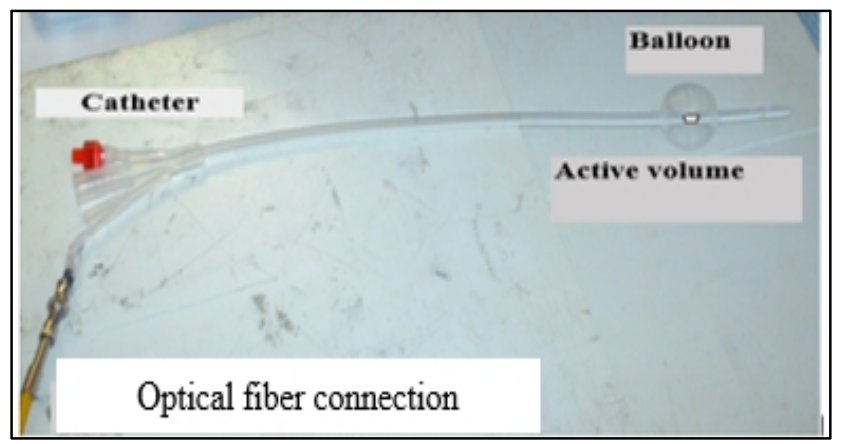

FIG. 3: A three channel bladder drain equipped with a GaN probe: the 3 channels are the following: drain, balloon inflation and $\mathrm{GaN}$ dosimeter probe insertion.

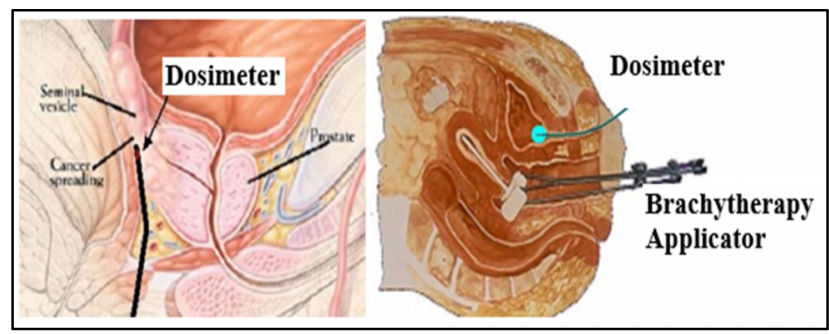

FIG. 4: Two suggested examples of the implantation of a dosimeter for pelvic cancer including prostate (left panel) and uterine cervix (right panel).

\section{Advantages, requirements and limitations}

The current implantable dosimeters have various advantages and limitations. The advantage of using an optical fiber coupled with a radio luminescent crystal is its ability to be implanted into, and retrieved from, a patient. This device can be inserted into natural cavities such as the rectum, head and neck cavities, or the bladder, using catheters, for example a bladder drain with a balloon, as shown in Figure 3. This requires a catheter having three channels: one for draining, one for inflating the balloon and one for inserting the optical fiber carrying the dosimeter. Such catheters currently exist for bladder hemorrhage washing. Figure 4 suggests possible applications of the $\mathrm{GaN}$ dosimeter for gynecological cancer using a device inserted into the bladder and for prostate cancer by way of an interstitial implantation into the perineum. However, most of these dosimeters need either electrical or optical connections; otherwise the dosimeter becomes rather large if it has to include wireless transmission technology. Moreover, optical fibers produce Cerenkov light, which must be eliminated, and needs shielding optical connections, a critical point. ${ }^{2}$

\section{Perspectives and new concepts}

As mentioned above, recent progress in radiotherapy technologies make it more and more possible to deliver higher doses over shorter times and fewer sessions. This makes each irradiation session more critical than previously. Thus, the opportunity to verify in real time the realization of each session is highly desirable to ensure the exact delivery of the planned treatment. The use of an implantable dosimeter appears to be an interesting solution; however present techniques have many drawbacks.

Further progress is necessary. The ideal dosimeter should have a very small size (on a nanometer scale), be easy to implant into the patient for real time in-situ dose measurements, have a low cost, be able to transmit the data via wireless communications, have no toxicity for the tissue and, why not, be resorbable. In terms of physical properties it should also have no energy or angular dependence, and not perturb the delivered dose. To our knowledge such a device is as yet far from existing.

The size of a nano detector is a critical point, because the size of a dosimeter must respect two criteria: firstly it should be large enough to detect enough events to produce a statistically reproducible measure of the dose in the range of expected use (in terms of type of beam and dose rate), and secondly it should be held in the tissues at the desired point within the target volume and not be displaced or removed by blood-flow or tissue movement due to pressure. M Beuve (2009) showed, using Monte Carlo simulation, that the local dose can be represented using a sensitive volume as large as $10 \mu \mathrm{m}$ in size in order to reduce significantly the statistical fluctuations in energy deposition, which could be deleterious for volumes as small as $10 \mathrm{~nm} .^{7,8}$

The main role for such a nano dosimeter would be to monitor the delivered dose for target volumes that include the tumor and organs at risk. One could imagine implanting a set 
of such devices at the most critical sites. Recently considerable progress has been made in the technology of nano materials and they are being integrated into medical applications for surgery, therapy, diagnostics, imaging and cancer treatments. We foresee that in the near future nano-technologies will enable us to produce nano-dosimeters based on nano materials.

\section{Conclusion}

The treatment of cancer using radiation therapy uses various modalities. Current techniques are becoming more and more sophisticated and can deliver higher doses per fraction in fewer fractions, giving the possibility to maximize the delivered dose to the tumor and to minimize the dose to surrounding tissues. Therefore the risk of inadequate or injurious sessions is an increasing challenge for optimal quality insurance. One solution could be the use of nanotechnology to provide new devices to monitor and measure the delivered dose in real time inside the target itself.

\section{Conflict of interest}

The authors declare that they have no conflicts of interest. The authors alone are responsible for the content and writing of the paper.

\section{Acknowledgements}

The authors acknowledge the financial support of the French National Research Agency (ANR-11-TECS-018). We thank France HADRON, the PRIMES "LabEx" for their interest and assistance; and Dr Alison Foote (Grenoble Clinical Research Center) for editing our manuscript.

\section{Reference}

1. Papanikolaou N, Battista J, Mackie T, Kappas C, Boyer A. Tissue inhomogeneity corrections for megavoltage photon beams. AAPM Report No 85; Task Group No. 65, 2004

2. Chaikh A, Balosso J. Should the dose prescription be readjusted when using tissues density corrections algorithms for radiation oncology? J Case Rep Onc Ther 2015; 1:118.

3. Chaikh A, Gaudu A, Balosso J. Monitoring methods for skin dose in interventional radiology. Int $J$ Cancer Ther Oncol2015; 3:03011.

4. Available from http://medicalphysicsweb.org/cws/article/research/ 25847
5. Andersen CE, Nielsen SK, Greilich S, et al. Characterization of a fiber-coupled Al2O3:C luminescence dosimetry system for online in vivo dose verification during 192Ir brachytherapy. Med Phys 2009; 36:708-18.

6. Chaikh A, Balosso J, Giraud JY, et al. Characterization of GaN dosimetry for $6 \mathrm{MV}$ photon beam in clinical conditions. Radiation measurements; 2014; 71:392-5.

7. Beuve M. Formalization and Theoretical Analysis of the Local Effect Model. Radiation research 2009; 172: 394-402.

8. Beuve M, Colliaux A, Dabli D, et al. Statistical effects of dose deposition in track-structure modelling of radiobiology efficiency. Nuclear Instruments and Methods in Physics Research Section B: Beam Interactions with Materials and Atoms 2009; 267: 983-8. 